\title{
Analysis of Long Noncoding RNA ZNF667-AS1 as a Potential Biomarker for Diagnosis and Prognosis of Glioma Patients
}

\author{
Qin Yuan, ${ }^{1}$ Chao Gao, ${ }^{2}$ Xiao-dong Lai, ${ }^{3}$ Liang-yi Chen $\mathbb{D}^{4},{ }^{4}$ and Tian-bao Lai $\mathbb{D}^{4}$ \\ ${ }^{1}$ Department of Oncology and Hematology, Hubei Provincial Hospital of Integrated Chinese and Western Medicine, Wuhan, \\ 430015 Hubei, China \\ ${ }^{2}$ Department of Oncology, First People's Hospital of Zaoyang, Zaoyang, 441200 Hubei, China \\ ${ }^{3}$ Fuzhou Medical College of Nanchang University, Fuzhou, 344000 Nanchang, China \\ ${ }^{4}$ Department of Neurology, Zhongshan Hospital Affiliated to Xiamen University, Xiamen, 361004 Fujian, China
}

Correspondence should be addressed to Liang-yi Chen; chenliangyix@163.com and Tian-bao Lai; dr.laitianbao@qq.com

Received 31 August 2020; Revised 15 September 2020; Accepted 19 October 2020; Published 17 November 2020

Academic Editor: Wen-Jun Tu

Copyright (C) 2020 Qin Yuan et al. This is an open access article distributed under the Creative Commons Attribution License, which permits unrestricted use, distribution, and reproduction in any medium, provided the original work is properly cited.

Objective. Long noncoding RNAs (lncRNAs) have been strongly associated with various types of cancer. The present study aimed at exploring the diagnostic and prognostic value of lncRNA Zinc finger protein 667-antisense RNA 1 (ZNF667-AS1) in glioma patients. Patients and Methods. The expressions of ZNF667-AS1 were detected in 155 glioma tissues and matched normal brain tissue samples by qRT-PCR. The receiver operating characteristic (ROC) curve was performed to estimate the diagnostic value of ZNF667-AS1. The association between the ZNF667-AS1 expression and clinicopathological characteristics was analyzed by the chi-square test. The Kaplan-Meier method was performed to determine the influence of the ZNF667-AS1 expression on the overall survival and disease-free survival of glioma patients. The Cox regression analysis was used to evaluate the effect of independent prognostic factors on survival outcome. Cell proliferation was measured by the respective cell counting Kit- 8 (CCK-8) assays. Results. We observed that ZNF667-AS1 was significantly upregulated in glioma tissues compared to normal tissue samples $(p<0.01)$. Higher levels of ZNF667-AS1 were positively associated with the WHO grade $(p=0.018)$ and KPS score $(p=0.008)$. ROC assays revealed that the high ZNF667-AS1 expression had an AUC value of 0.8541 (95\% CI: 0.8148 to 0.8934) for glioma. Survival data revealed that glioma patients in the high ZNF667-AS1 expression group had significantly shorter 5-year overall survival $(p=0.0026)$ and disease-free survival $(p=0.0005)$ time than those in the low ZNF667-AS1 expression group. Moreover, multivariate analyses confirmed that the ZNF667-AS1 expression was an independent predictor of the overall survival and disease-free survival for glioma patients. Functionally, we found that knockdown of ZNF667-AS1 suppressed the proliferation of glioma cells. Conclusions. Our results suggest that ZNF667-AS1 could be used as a potential diagnostic and prognostic biomarker in glioma.

\section{Introduction}

Human gliomas refer to the commonest malignant tumors of the core nervous mechanism, achieving 6/100,000 incidence each year globally [1]. Malignant glioma exhibits aggressiveness, high infiltration, and resistance to normal therapies [2]. It is noteworthy that glioblastoma multiform (GBM), a particular glioma kind, exhibits the highest aggressiveness for the rapid growing process and frequent spread to close brain-related tissues [3]. Though the overall treating condition of brain gliomas continues to progress, such malig- nancy outcome is not optimized significantly $[4,5]$. Thus, emerging molecular markers should be developed for improving gliomas classifying process, patient prognosis, and treating plans.

Over the past few years, according to research with various overall genome methods, e.g., the ENCODE program, most mammalian genome receives transcription, whereas only $1.2 \%$ of the mentioned transcripts suggest the genes coding protein $[6,7]$. Long noncoding RNAs (lncRNAs) refer to a sequence of transcripts with over $200 \mathrm{nt}$ exhibiting slight or no ability to the code protein and critically impact a 
wide range of cellular processes, e.g., splicing, $\mathrm{X}$ chromosome inactivation, epigenetic controls, and gene transcription regulation $[8,9]$. Numerous lncRNAs suggested aberrant expression in various cancer types, and several lncRNAs with dysregulation become oncogenes or tumor-suppressing elements under special cases $[10,11]$. For instance, lncRNA NFIA-AS2 was shown to be highly expressed in glioma and promoted the proliferation and migration of glioma cells via modulating the miRNA-655-3p/ZFX axis [12]. LncRNA RNCR3 was shown to promote the proliferation and metastasis of glioma cells via regulating the Akt/GSK-3 $\beta$ pathway [13]. In addition, more and more lncRNAs were identified to be potential diagnostic and prognostic biomarkers for various tumor patients, including glioma [14-17]. Although several studies have investigated lncRNAs in glioma, their completely biological roles and clinical significance are still obscure.

LncRNA Zinc finger protein 667-antisense RNA 1 (ZNF667-AS1) in chromosome 19q13.43 of human was recently identified as tumor-related lncRNA. Previously, several studies have reported that ZNF667-AS1 was abnormally expressed in several tumor specimens and cells by the use of RT-PCR and high-throughput sequencing [18-20]. In addition, its tumor-related functions acting as tumor promotors or antioncogenes have been reported in nasopharyngeal carcinoma, esophageal squamous cell carcinoma, and cervical cancer [21-23]. However, whether ZNF667-AS1 also displayed an abnormal expression in glioma had not been investigated. Here, its expressing pattern and clinical significance in glioma patients are to be primarily explored.

\section{Patients and Methods}

2.1. Patient Data and Tissue Samples. Patients with glioma admitted to the oncology from June 2012 to August 2015 were drawn from the hospital tumor registry. The 155 enrolled patients were aged from 29 to 65 years, with a median age of 42 years. Histological diagnoses were made according to the 2007 WHO classification. 141 glioma patients completed follow-up, and the rate of loss to follow-up was 9\%. All the patients did not receive prior anticancer treatment.

The flow chart in the section of study patients was shown in Figure 1. This study proved overall samples by pathologyrelated tests and incubated them in liquid nitrogen to achieve the subsequent overall RNA extracting process. The clinical and pathological data for the patients are reported in Table 1 . The present study was ethically approved by the Research Ethics Committee of the Hubei Provincial Hospital of Integrated Chinese and Western Medicine (number: 20180056), and written informed consent was acquired from patient.

2.2. Cell Culture and siRNA Transfection. Human glioma cell lines, LN118, SHG44, U87, U251, and A172, were obtained from Cell Bank of Chinese Academy of Sciences (Pudong, Shanghai, China). The cells were grown in the Roswell Park Memorial Institute (RPMI) 1640 medium, supplemented with $10 \%$ fetal bovine serum (FBS) (Yunshan Technology, Haidian, Beijing, China), $100 \mathrm{U} / \mathrm{ml}$ penicillin $\mathrm{G}$ (Yunshan Technology, Haidian, Beijing, China), and $100 \mu \mathrm{g} / \mathrm{ml}$ strepto- mycin (Sigma, Suzhou, Jiangsu, China). Normal human astrocytes (NHA) were obtained from Weihui Biot (Hangzhou, Zhejiang, China) and were grown in Dulbecco's Modified Eagle's Medium with high glucose and sodium pyruvate.

The siRNAs used to knockdown ZNF667-AS1 were purchased from GEMA GENE (Pudong, Shanghai, China). The siRNA sequences were as follows: si-ZNF667-AS1: CTAC CACAGCTTCCATG; si-ZNF667-AS1-2: GCCCACTGTAT TCAACA. Cell transfection was conducted by using the LipofectamineTM 2000 transfection reagent (Invitrogen, Guangzhou, Guandong, China) according to the manufacturer's instructions. After transfection for $24 \mathrm{~h}$, the knockdown efficiency was evaluated by RT-PCR.

2.3. Reverse Transcription and $q P C R$ Analyses. Total RNA from glioma specimens and nontumor specimens was extracted with the Trizol reagent (Takara, Kunshan, Jiangsu, China) by complying with the plan of the producer. RNA concentration was ascertained; this study adopted $100 \mu \mathrm{g}$ of RNA during reverse transcription. Based on an ABI 7900 Real-Time PCR System, this study conducted qPCR with the plan below: $95^{\circ} \mathrm{C}$ for $3 \mathrm{~min}$., 40 cycles of $95^{\circ} \mathrm{C}$ for $15 \mathrm{sec}$., $60^{\circ} \mathrm{C}$ for $15 \mathrm{sec}$., and $72^{\circ} \mathrm{C}$ for $30 \mathrm{sec}$. The comparative expressing level of ZNF667-AS1 underwent the normalization to GAPDH. The primers included ZNF667-AS1 forward, $5^{\prime}$-GGTCCACTTCACGCACTTGC-3'; ZNF667-AS1 reverse, 5'-ACCATTCGAACTTGGCTACA-3'; GAPDH forward, 5' -GTCAACGGATTTGGTCTGTA-3'; GAPDH reverse, $5^{\prime}$-AGTCTTCTGGGTGGCAGTG- $3^{\prime}$. Using the $2^{-}$ $\Delta \Delta \mathrm{Ct}$ approach, the relative expression conditions of the gene of interest were obtained. qRT-PCR reaction processes were overall performed 3 times.

2.4. Cell Proliferation Assays. For the cell proliferation analysis, 300 living cells (A172 and LN118 cells) were transfected with either si-ZNF667-AS1-1, ZNF667-AS1-2, or a scrambled control and plated onto 96-well plates. Every $24 \mathrm{~h}$, the CCK- 8 solution $(10 \mu \mathrm{l})$ was added to three randomly selected wells, and the cultures were incubated at $37^{\circ} \mathrm{C}$ for $90 \mathrm{~min}$. The absorbance was measured at $450 \mathrm{~nm}$ using a microplate reader.

2.5. Statistical Analysis. Statistical analysis was conducted with SPSS version 13.0 software (SPSS Inc, Chicago, IL, USA). With the Student's $t$-test, the diversifications of two cohorts were discussed. The relationship between the ZNF667-AS1-expressing level and clinical-pathological characteristics was analyzed with the chi-square test. Based on the Kaplan-Meier approach, the overall survival (OS) and disease-free survival (DFS) ratios were obtained with the log-rank experiment adopted to draw the comparing process. Multivariate Cox regression analyses were carried out for analyzing the survival information. A $p$ value $<0.05$ was suggested to exhibit statistical significance.

\section{Results}

3.1. ZNF667-AS1 Was Upregulated in Glioma. To determine the possible functions of ZNF667-AS1 in glioma, we enrolled 


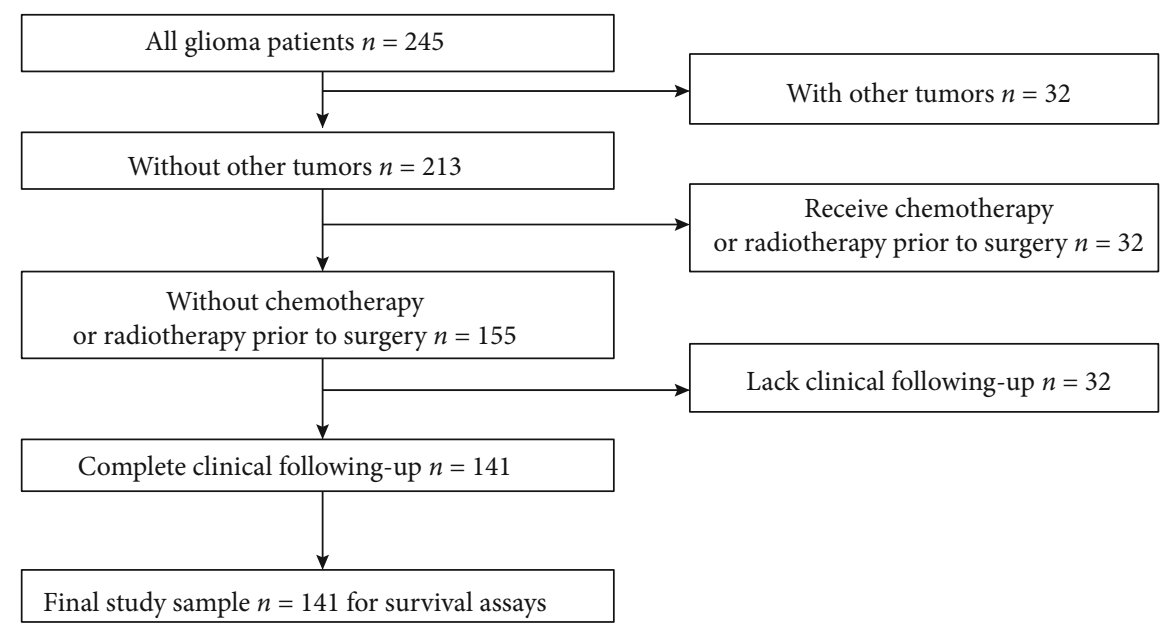

FIgURE 1: Flow chart in the selection of study patients.

TABLE 1: The association between the ZNF667-AS1 expression and different clinicopathological features of 155 human gliomas.

\begin{tabular}{|c|c|c|c|c|}
\hline \multirow[t]{2}{*}{ Parameter } & \multirow[t]{2}{*}{ No. of cases } & \multicolumn{2}{|c|}{$\begin{array}{c}\text { ZNF667-AS1 } \\
\text { expression }\end{array}$} & \multirow[t]{2}{*}{$p$ value } \\
\hline & & High & Low & \\
\hline Age & & & & 0.369 \\
\hline$<50$ & 82 & 39 & 43 & \\
\hline$\geq 50$ & 73 & 40 & 33 & \\
\hline Gender & & & & 0.394 \\
\hline Male & 93 & 50 & 43 & \\
\hline Female & 62 & 29 & 33 & \\
\hline WHO grade & & & & 0.005 \\
\hline I-II & 108 & 47 & 61 & \\
\hline III-IV & 47 & 32 & 15 & \\
\hline KPS score & & & & 0.008 \\
\hline$<80$ & 57 & 37 & 20 & \\
\hline$\geq 80$ & 98 & 42 & 56 & \\
\hline Tumor size & & & & 0.145 \\
\hline$<3 \mathrm{~cm}$ & 95 & 44 & 51 & \\
\hline$\geq 3 \mathrm{~cm}$ & 60 & 35 & 25 & \\
\hline
\end{tabular}

155 glioma patients and performed RT-PCR to examine the expression of ZNF667-AS1 in a total of 155 paired glioma tissues and corresponding nontumor tissues. As shown in Figure 2(a), we observed that the ZNF667-AS1 expression was distinctly increased in glioma specimens compared to matched nontumor specimens $(p<0.01)$. Besides, we also observed that glioma specimens with advanced stages exhibited a higher level of ZNF667-AS1 than those with early stages $(p<0.01$, Figure 2(b)). Overall, our findings suggested ZNF667-AS1 as a functional regulator in glioma progression.

3.2. The Diagnostic Significance of the Overexpression of ZNF667-AS1 in Glioma. Previous studies have revealed several functional lncRNA that displayed a diagnostic value in glioma patients. Then, we performed ROC assays which showed that the high ZNF667-AS1 expression had an AUC value of 0.8541 ( $95 \%$ CI: 0.8148 to 0.8934 ) for glioma (Figure 3(a)). The sensitivity and specificity of ZNF667-AS1 expressions for distinguishing glioma samples from normal samples were $68.22 \% / 84.57 \%$. In addition, we also observed that ZNF667-AS1 could be used as a molecular marker for distinguishing glioma specimens with advanced stages from glioma specimens with early stages with an AUC of 0.7742 (95\% CI: 0.6990-0.8494; $p<0.01$, Figure 3(b)). Our findings suggested ZNF667-AS1 as an early diagnosis indicator for glioma patients.

3.3. Correlations between ZNF667-AS1 and Clinical Features of Glioma. For better understanding of the clinical relevance of the ZNF667-AS1 expression in glioma, the 155 glioma patients were split as a high expression cohort $(n=79)$ and a low expression cohort $(n=76)$, complying with the median expressing condition of ZNF667-AS1 (5.62) in all glioma samples. Then, our group determined the possible association between ZNF667-AS1 levels and several clinical factors using the chi-square test which suggested that the high ZNF667-AS1 expression was associated with the WHO grade $(p=0.005)$ and KPS score $(p=0.008$, Table 1$)$. However, there were no significant correlations of the ZNF667-AS1 expression with other clinical features $(p>0.05)$.

3.4. The Possible Prognostic Values of the ZNF667-AS1 Expression in Glioma. To further explore whether ZNF667AS1 may display a positive influence on the survivals of glioma patients, we collected the survival data of all 141 patients who completed five-year follow-up, which were analyzed using the Kaplan-Meier survival curves. Interestingly, our results revealed that patients with the high expression of ZNF667-AS1 had shorter OS $(p<0.0026$; Figure 4(a)) and DFS $(p<0.005$; Figure $4(\mathrm{~b}))$ as compared with the ZNF667-AS1 low group. More importantly, in a multivariate Cox model, we demonstrated that the ZNF667-AS1 expression was an independent poor prognostic factor for both 5 -year OS (HR $=2.897,95 \%$ CI: 1.365 - 


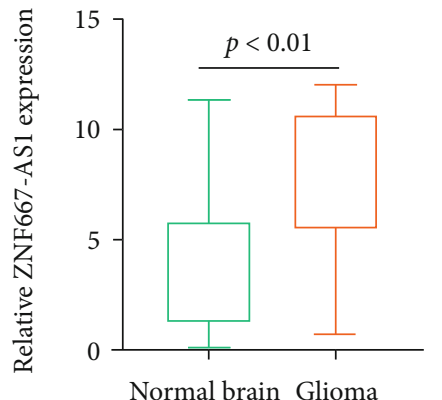

(a)

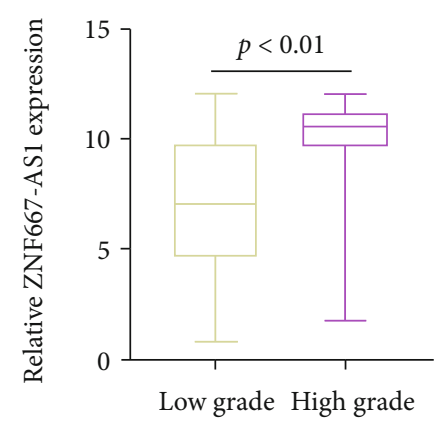

(b)

FIGURE 2: The ZNF667-AS1 expression is increased in glioma tissues. (a) The relative expression of ZNF667-AS1 in paired tumor and nontumor tissues $(n=155)$ by RT-PCR. The expression of ZNF667-AS1 in different tumor grades.

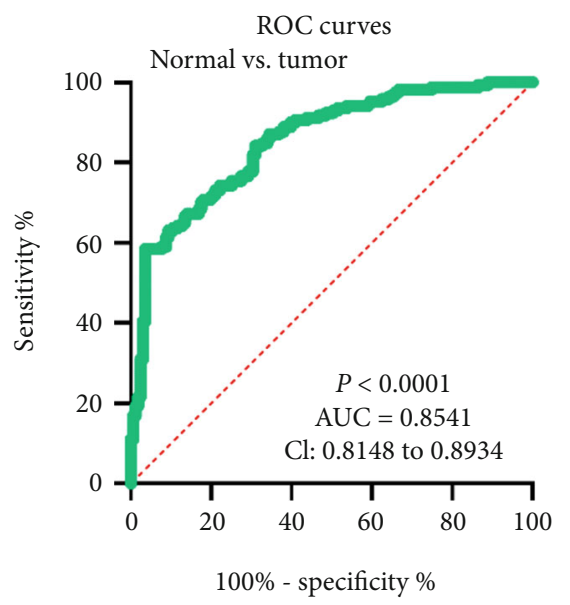

(a)

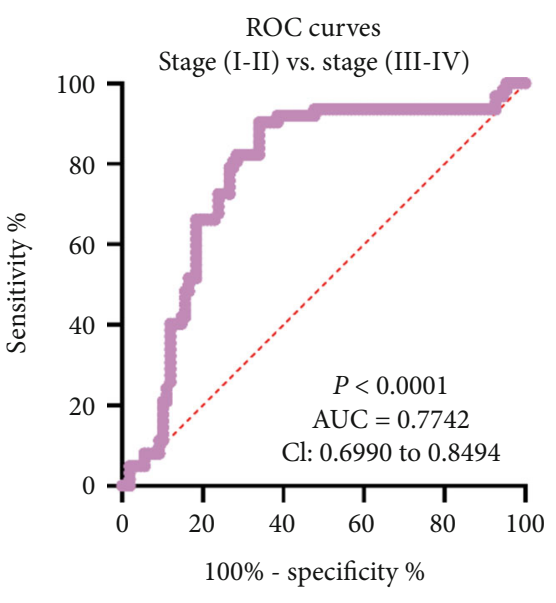

(b)

FIGURE 3: ZNF667-AS1 may be a potential diagnostic biomarker for glioma patients. (a) The ROC curve for the diagnostic value of ZNF667AS1 in distinguishing glioma specimens from nontumor brain specimens. (b) The ROC curve for the diagnostic value of ZNF667-AS1 in distinguishing glioma specimens with stages (I-II) from glioma specimens with stages (III-IV).

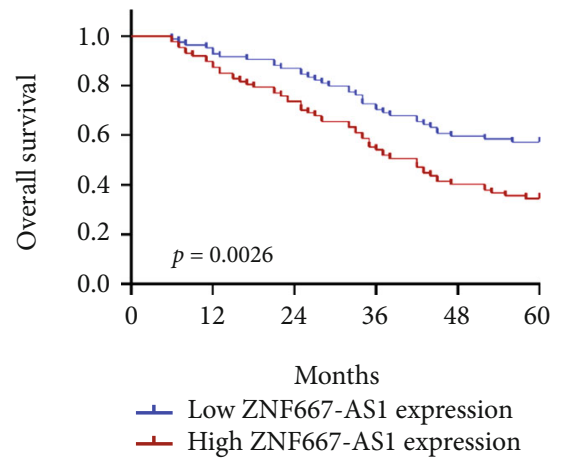

(a)

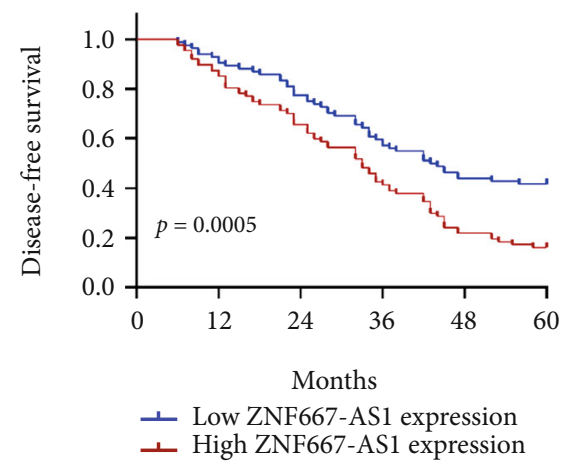

(b)

Figure 4: Kaplan-Meier curves of the overall survival (a) and disease-free survival (b) of 155 glioma patients.

$4.784, p=0.008)$ and 5-year DFS $(\mathrm{HR}=3.019, \mathrm{CI}=1.414-$ $4.899, p=0.005)$ in glioma (Table 2 ).

3.5. Effects of ZNF667-AS1 on Proliferation of Glioma Cells. To analyze the possible function of ZNF667-AS1 on the proliferation of glioma cell, we firstly examine the expression of ZNF667-AS1 in five glioma cells, finding that ZNF667-AS1 was highly expressed in five glioma cells compared to NHA (Figure 5(a)). Because A172 and LN118 cells exhibited a higher level of ZNF667-AS1, we chose them for the following experiments. Real-time PCR was performed to confirm the successful knockdown of ZNF667-AS1 in A172 and LN118 cells after the transfection of si-ZNF667-AS1-1 and siZNF667-AS1-2 (Figure 5(b)). CCK-8 assays revealed that 
TABLE 2: Multivariate analysis of the overall survival and disease-free survival in glioma patients.

\begin{tabular}{|c|c|c|c|c|c|c|}
\hline \multirow{2}{*}{ Variables } & \multicolumn{3}{|c|}{ Overall survival } & \multicolumn{3}{|c|}{ Disease free survival } \\
\hline & HR & $95 \% \mathrm{CI}$ & $p$ value & HR & $95 \%$ CI & $p$ value \\
\hline Age & 0.882 & $0.452-1.342$ & 0.233 & 1.113 & $0.673-1.732$ & 0.143 \\
\hline Gender & 1.442 & $0.642-2.104$ & 0.342 & 1.632 & $0.773-2.341$ & 0.219 \\
\hline WHO grade & 2.893 & $1.324-4.872$ & 0.009 & 3.014 & $1.432-5.118$ & 0.005 \\
\hline KPS score & 2.782 & $1.423-4.563$ & 0.013 & 2.964 & $1.395-4.895$ & 0.007 \\
\hline Tumor size & 1.237 & $0.672-1.897$ & 0.213 & 1.427 & $0.875-2.137$ & 0.136 \\
\hline ZNF667-AS1 expression & 2.897 & $1.365-4.784$ & 0.008 & 3.019 & $1.414-4.899$ & 0.005 \\
\hline
\end{tabular}

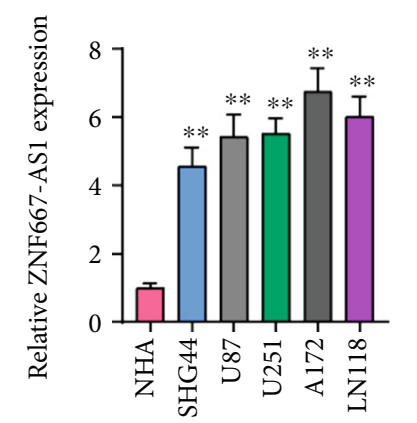

(a)

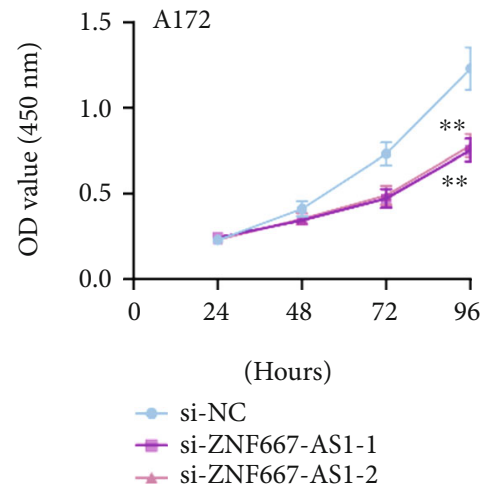

(c)

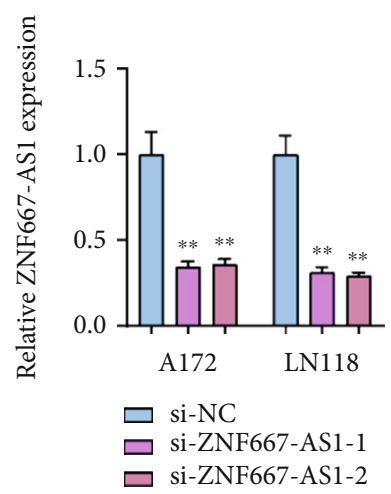

(b)

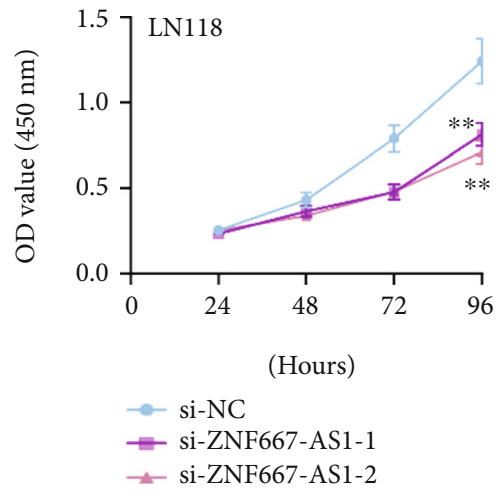

(d)

FIGURE 5: Knockdown of ZNF667-AS1 silencing inhibits the proliferation of A172 and LN118 cells. (a) RT-PCR for the demonstration of the ZNF667-AS1 expression in five glioma cells and NHA. (b) qPCR analyses of ZNF667-AS1 levels following treatment of A172 and LN118 cells with si-ZNF667-AS1-1, ZNF667-AS1-2, or si-NC. (c, d) The MTT assay was performed to determine the proliferation of A172 and LN118. ${ }^{* *} p<0.05$.

knockdown of ZNF667-AS1 significantly suppressed cell proliferation in A172 and LN118 cells (Figures 5(c) and 5(d)). Overall, our findings suggested that ZNF667-AS1 served as a tumor promotor in glioma.

\section{Discussion}

Glioma is the most common intracranial malignant tumor in humans $[1,24]$. Highly glioma presents unique challenges due to its tendency to proliferate and invade tissues. Malignant gliomas, including glioblastomas, with radiotherapy and temozolomide have only a small survival benefit [25, 26]. The sensitive biomarkers were very important for the treatments of tumor patients $[27,28]$. However, none of the currently identified biomarkers are sensitive or specific enough for reliable glioma screening in clinical settings. In recent years, growing studies have indicated that some functional lncRNAs could be used as novel biomarkers for prognosis, survival, and responses to the treating process that paved a novel wave of research into molecular markers of glioma $[29,30]$. In this study, we forced on a novel gliomarelated lncRNA ZNF667-AS1.

In recent years, several studies have reported the dysregulated expression of ZNF667-AS1 and its possible effects in several tumors. For instance, Chen et al. [21] reported that the expression of ZNF667-AS1 was distinctly decreased in 
nasopharyngeal carcinoma specimens and cell lines. Functionally, the overexpression of ZNF667-AS1 was shown to suppress the proliferation of nasopharyngeal carcinoma cells via increasing the ABLIM1 expression via adsorbing miR-1290. Li et al. [23] showed that ZNF667-AS1 was significantly highly expressed in cervical cancer, and its low levels suggested a poor prognosis of cervical cancer patients. In vitro and in vivo assays indicated that the forced expression of ZNF667-AS1 inhibited the proliferation and metastasis through counteracting the miR-93-3p-dependent PEG3 suppression. Moreover, ZNF667-AS1 was also shown to suppress the inflammatory response, promoting the recovery of spinal cord injury via suppressing the JAK-STAT pathway [31]. The negatively regulatory association between ZNF667AS1 and JAK-STAT pathway highlighted its potential effects acting as tumor suppressors in tumors due to the important effects of the JAK-STAT pathway in tumor progression. All previous findings suggested ZNF667-AS1 may be a critical regulator in several tumor kinds. However, the expressing and clinical value of ZNF667-AS1 in glioma remained unclear.

Here, the expressing condition of ZNF667-AS1 in 155 glioma patients was first examined, confirming that the ZNF667-AS1 expression was distinctly upregulated in glioma specimens in comparison with matched nontumor brain tissues, which was inconsistent with its expression trend in other several tumors. Then, we determined the diagnostic value in glioma patients and found that ZNF667-AS1 in tissues effectively differentiated glioma tissues from nontumor specimens with an area under the ROC curves (AUC) of 0.8541. The diagnostic value of the ZNF667-AS1 expression was also confirmed in patients with different stages. Then, we analyzed the clinical significance of the ZNF667-AS1 expression, finding that the high ZNF667-AS1 expression was associated with the KPS score and advanced WHO grade, suggesting it that acted as a tumor promotor in clinical progression of glioma. Moreover, after analyzing the survival data with five-year following-up, we confirmed that patients with high ZNF667-AS1 expressions exhibited a longer OS and DFS than those with low ZNF667-AS1 expressions. Finally, the results of the multivariate study verified ZNF667-AS1 a single predictor for OS and DFS in glioma patients. On the other hand, we also performed CCK-8 assays to explore the tumor-related effects of ZNF667-AS1 on glioma cells, confirming that knockdown of ZNF667-AS1 suppressed the proliferation of glioma cells. Our findings suggested ZNF667-AS1 as a tumor promotor in glioma progression.

Some limitations of this study should be noted. First, because of the small number of patients analyzed in our research, more patients for clinical experiments are required to confirm our findings. Second, we fail to determine whether the serum ZNF667-AS1 expression was dysregulated in glioma patients. The serum biomarkers are very important for the monitor of the therapy response in real-time. Third, the potential effects of ZNF667-AS1 on the metastasis ability and in vivo assays are not performed. In the future, we plan to more fully describe the mechanisms and relationships with lncRNAs related to glioma biomarkers.

\section{Conclusions}

The results revealed initially that ZNF667-AS1 may represent a valuable independent prognostic indicator for glioma. Further study to discover the molecular mechanism of ZNF667AS1 about tumor migration and invasion is currently in progress.

\section{Data Availability}

The data used to support the findings of this study are available from the corresponding author upon request.

\section{Conflicts of Interest}

The authors declare no conflicts of interest.

\section{Authors' Contributions}

Qin Yuan and Chao Gao are co-first authors.

\section{Acknowledgments}

This study was supported by the Fujian Provincial Health and Family Planning Youth Scientific Research Project (No. 2016-2-66).

\section{References}

[1] Q. T. Ostrom, L. Bauchet, F. G. Davis et al., "The epidemiology of glioma in adults: a "state of the science" review," NeuroOncology, vol. 16, no. 7, pp. 896-913, 2014.

[2] S. A. Grimm and M. C. Chamberlain, "Brainstem glioma: a review," Current Neurology and Neuroscience Reports, vol. 13, no. 5, 2013.

[3] R. Chen, M. Smith-Cohn, A. L. Cohen, and H. Colman, "Glioma subclassifications and their clinical significance," $\mathrm{Neu}$ rotherapeutics, vol. 14, no. 2, pp. 284-297, 2017.

[4] N. A. Bush, S. M. Chang, and M. S. Berger, "Current and future strategies for treatment of glioma," Neurosurgical Review, vol. 40, no. 1, pp. 1-14, 2017.

[5] S. L. Hervey-Jumper and M. S. Berger, "Insular glioma surgery: an evolution of thought and practice," Journal of Neurosurgery, vol. 130, no. 1, pp. 9-16, 2019.

[6] L. Liu, Q. Wang, Z. Qiu et al., "Noncoding RNAs: the shot callers in tumor immune escape," Signal Transduction and Targeted Therapy, vol. 5, no. 1, 2020.

[7] M. Matsui and D. R. Corey, "Non-coding RNAs as drug targets," Nature Reviews Drug Discovery, vol. 16, no. 3, pp. 167-179, 2017.

[8] S. Jathar, V. Kumar, J. Srivastava, and V. Tripathi, “Technological developments in lncRNA biology," Advances in Experimental Medicine and Biology, vol. 1008, pp. 283-323, 2017.

[9] M. M. Kumar and R. Goyal, "LncRNA as a therapeutic target for angiogenesis," Current Topics in Medicinal Chemistry, vol. 17, no. 15, pp. 1750-1757, 2017.

[10] G. Romano, M. Saviana, P. Le et al., "Non-coding RNA editing in cancer pathogenesis," Cancers, vol. 12, no. 7, article 1845, 2020. 
[11] F. Ferrè, A. Colantoni, and M. Helmer-Citterich, "Revealing protein-lncRNA interaction," Briefings in Bioinformatics, vol. 17, no. 1, pp. 106-116, 2016.

[12] J. Xin, Y. H. Zhao, X. Y. Zhang, and L. Q. Tian, "LncRNA NFIA-AS2 promotes glioma progression through modulating the miR-655-3p/ZFX axis," Human Cell, vol. 33, no. 4, pp. 1273-1280, 2020.

[13] B. Zhu, S. Zhang, N. Meng, H. Zhang, S. Yuan, and J. Zhang, "Long non-coding RNA RNCR3 promotes glioma progression involving the Akt/GSK-3 $\beta$ pathway," Oncology Letters, vol. 18, no. 6, pp. 6315-6322, 2019.

[14] A. M. Schmitt and H. Y. Chang, "Long noncoding RNAs in cancer pathways," Cancer Cell, vol. 29, no. 4, pp. 452-463, 2016.

[15] J. Li, Z. Li, W. Zheng et al., "LncRNA-ATB: an indispensable cancer-related long noncoding RNA," Cell proliferation, vol. 50, no. 6, article e12381, 2017.

[16] J. Song, X. Chen, Q. Tian et al., "The value of lncRNA GHET1 as a prognostic factor for survival of Chinese cancer outcome: a meta-analysis," Disease Markers, vol. 2019, Article ID 5824190, 7 pages, 2019.

[17] J. Huang, Y. Zheng, X. Xiao et al., “A circulating long noncoding RNA panel serves as a diagnostic marker for hepatocellular carcinoma," Disease Markers, vol. 2020, Article ID 5417598, 12 pages, 2020.

[18] L. Vrba and B. W. Futscher, "Epigenetic silencing of lncRNA MORT in 16 TCGA cancer types," F1000Research, vol. 7, 2018.

[19] L. Vrba and B. W. Futscher, "Epigenetic silencing of MORT Is an early event in cancer and is associated with luminal, receptor positive breast tumor subtypes," Journal of breast cancer, vol. 20, no. 2, pp. 198-202, 2017.

[20] L. Vrba, J. C. Garbe, M. R. Stampfer, and B. W. Futscher, “A lincRNA connected to cell mortality and epigeneticallysilenced in most common human cancers," Epigenetics, vol. 10, no. 11, pp. 1074-1083, 2015.

[21] X. Chen, Y. Huang, D. Shi et al., "LncRNA ZNF667-AS1 Promotes ABLIM1 Expression by Adsorbing microRNA-1290 to Suppress Nasopharyngeal Carcinoma Cell Progression," OncoTargets and Therapy, vol. 13, pp. 4397-4409, 2020.

[22] Z. Dong, S. Li, X. Wu et al., "Aberrant hypermethylationmediated downregulation of antisense lncRNA ZNF667-AS1 and its sense gene ZNF667 correlate with progression and prognosis of esophageal squamous cell carcinoma," Cell Death \& Disease, vol. 10, no. 12, 2019.

[23] Y. J. Li, Z. Yang, Y. Y. Wang, and Y. Wang, "Long noncoding RNA ZNF667-AS1 reduces tumor invasion and metastasis in cervical cancer by counteracting microRNA-93-3p-dependent PEG3 downregulation," Molecular oncology, vol. 13, no. 11, pp. 2375-2392, 2019.

[24] Z. Zhao, K. N. Zhang, R. C. Chai et al., "ADAMTSL4, a secreted glycoprotein Is a Novel Immune-Related Biomarker for Primary Glioblastoma Multiforme," Disease markers, vol. 2019, Article ID 1802620, 12 pages, 2019.

[25] S. Lapointe, A. Perry, and N. A. Butowski, "Primary brain tumours in adults," Lancet (London, England), vol. 392, no. 10145 , pp. 432-446, 2018.

[26] D. Bready and D. G. Placantonakis, "Molecular pathogenesis of low-grade glioma," Neurosurgery Clinics of North America, vol. 30, no. 1, pp. 17-25, 2019.

[27] R. X. Geng, N. Li, Y. Xu et al., "Identification of core biomarkers associated with outcome in glioma: evidence from bioinformatics analysis," Disease markers, vol. 2018, Article ID 3215958, 16 pages, 2018.

[28] F. Yuan, L. Yi, L. Hai et al., "Identification of key pathways and genes in the Orai2 mediated classical and mesenchymal subtype of glioblastoma by bioinformatic analyses," Disease markers, vol. 2019, Article ID 7049294, 13 pages, 2019.

[29] M. Velázquez-Flores, J. M. Rodríguez-Corona, J. E. LópezAguilar et al., "Noncoding RNAs as potential biomarkers for DIPG diagnosis and prognosis: XIST and XIST-210 involvement," Clinical and Translational Oncology, vol. 22, 2020.

[30] A. Zottel, N. Šamec, A. Videtič Paska, and I. Jovčevska, "Coding of glioblastoma progression and therapy resistance through long noncoding RNAs," Cancers, vol. 12, no. 7, article $1842,2020$.

[31] J. W. Li, Y. Kuang, L. Chen, and J. F. Wang, "LncRNA ZNF667-AS1 inhibits inflammatory response and promotes recovery of spinal cord injury via suppressing JAK-STAT pathway," European review for medical and pharmacological sciences, vol. 22, no. 22, pp. 7614-7620, 2018. 